\title{
Low-Mass Extremely Metal-Poor Stellar Models: Yields, Uncertainties and the Galactic Halo Stars
}

\author{
Simon W. Campbell ${ }^{1,2}$ and J. C. Lattanzio ${ }^{1}$ \\ ${ }^{1}$ Academia Sinica Institute of Astronomy and Astrophysics, \\ P.O. Box 23-141, Taipei, Taiwan 10617 \\ email: simcam@asiaa.sinica.edu.tw \\ ${ }^{2}$ Centre for Stellar and Planetary Astrophysics, School of Mathematical Sciences, \\ Monash University, Melbourne, Australia 3800 \\ email: john.lattanzio@sci.monash.edu.au
}

\begin{abstract}
We have calculated a set of low-mass $\left(0.85 M_{\odot} \leqslant M \leqslant 3.0 M_{\odot}\right)$ zero metallicity and extremely metal-poor $(-6.5 \leqslant[\mathrm{Fe} / \mathrm{H}] \leqslant-3.0)$ stellar models, including nucleosynthetic yields for 74 species. As far as we are aware these are the first detailed yields in the mass and metallicity range considered. Due to the difficulty in modelling such stars the yields naturally contain numerous uncertainties, and thus present interesting challenges for future stellar modelling. We briefly present some results in the context of the Galactic Halo star observations, and also discuss qualitatively some of the uncertainties in the modelling. We conclude by suggesting that much work is still necessary in this research area. For example, multidimensional fluid dynamics models are needed to simulate the violent proton ingestion events that occur during the core $\mathrm{He}$ flash and early TPAGB, observations and theory of mass loss at low metallicities are needed, the effects of reaction rate uncertainties need to be quantified, and low temperature opacities variable in carbon (and nitrogen) need to be included in the models.
\end{abstract}

Keywords. stars: AGB and post-AGB, stars: evolution

\section{Introduction}

The discovery of extremely metal-poor stars (EMPs) in the Galactic Halo has renewed interest in the theoretical modelling of Population III and low-metallicity stars (eg. Fujimoto et al. 1990, Cassisi et al. 1996, Siess et al. 2002, Schlattl et al. 2002, Picardi et al. 2004, Suda et al. 2004). Most of these stars show abundances that conform to a simple Galactic chemical evolution line (see Figure 1). However a subset of the EMP stars have been observed to contain large amounts of carbon. These C-rich EMPs (CEMPs) make up a large proportion of the EMP population $(\sim 10 \rightarrow 20 \%$; see eg. Beers \& Christlieb 2005). This population is also highlighted in Figure 1. Apart from carbon the EMP stars also display variation in a range of other elements (see Beers \& Christlieb 2005 for a review of the observations). A number of theories have been proposed to explain the various patterns, ranging from pre-formation pollution via Pop III SNe (eg. Shigeyama \& Tsujimoto 1998, Limongi et al. 2003) to self-pollution through peculiar evolutionary events (eg. Fujimoto et al. 2000, Weiss et al. 2004) to binary mass transfer (eg. Suda et al. 2004).

In the current study we have undertaken a broad exploration of EMP stellar evolution and nucleosynthesis in the low and intermediate mass regime $\left(0.85 M_{\odot} \leqslant M \leqslant 3.0 M_{\odot}\right)$. Our study expands on the previous work in the field. In particular it includes full evolutionary calculations from ZAMS to the end of the thermally-pulsing AGB phase 
(TPAGB), as well as chemical yields for 74 nuclear species. With this homogeneous set of models we hope to shed some light on whether or not 1D stellar models can explain some of the EMP halo star observations.

\section{Method}

Our simulations were performed utilising two numerical codes - a stellar structure code and post-process nucleosynthesis code.

The stellar structure code used was the Monash version of the Monash/Mount Stromlo stellar evolution code (MONSTAR; see eg. Wood \& Zarro 1981, Frost \& Lattanzio 1996). The code is largely a standard 1D code that utilises the Henyey-matrix method (a modified Newton-Raphson method) for solving the stellar structure equations. Opacities have been updated to those from Iglesias \& Rogers (1996) (for mid-range temperatures) and Ferguson et al. (2005) (for low temperatures). For the present study the instantaneous convective mixing routine was replaced by a time-dependent (diffusive) mixing routine (similar to that described by Meynet et al. 2004). This change was necessary due to the violent evolutionary events that occur in models of $Z=0$ and EMP stars. Convective boundaries were always defined by the Schwarzschild criterion - ie. the search for a neutral convective boundary was not performed and no overshoot was applied. In terms of the resultant chemical yields, the extent of convective zones in this study could thus be considered as conservative, since any extension of the Schwarzschild boundary would lead to even more pollution.

The nucleosynthesis calculations were made with the Monash Stellar Nucleosynthesis code (MONSN), a post-process code. As input it takes the key structural properties of each hydrostatic model from the MONSTAR code (eg. density, temperature, convective velocities). It solves a network of 506 nuclear reactions involving 74 nuclear species (see eg. Cannon 1993, Lattanzio et al. 1996, Lugaro et al. 2004 for more details on this code). Our grid of models includes the following masses: $M=0.85,1.0,2.0,3.0 M_{\odot}$ and metallicities: $[\mathrm{Fe} / \mathrm{H}]=-6.5,-5.45,-4.0,-3.0$, plus $Z=0$.

\section{Brief Results: Carbon}

\subsection{Comparisons with Observations}

In Figure 1 we compare the carbon yields from our entire grid of models with the observed $[\mathrm{C} / \mathrm{Fe}]$ abundances in EMP halo stars. It can be seen that the yields from our models are all $\mathrm{C}$-rich. The reason for this is that the low-mass $\left(M=0.85 \& 1.0 M_{\odot}\right)$ models that would not normally have C-rich yields, due to a lack of third dredge-up (TDU, represented by green dots in 1), undergo proton ingestion episodes (PIEs) and subsequent dredge-up of material that has experienced He burning. In these models this event occurs during the core He flash at the tip of the RGB. In the intermediate mass models $\left(M=2.0,3.0 M_{\odot}\right)$ a similar event occurs at the beginning of the TPAGB phase (this time involving proton ingestion into the AGB He shell). In both cases a double spike in luminosity occurs (from the normal He flashes plus the PIEs). For this reason we refer to these events as 'Dual Core Flashes' (DCFs, at the RGB tip, red crosses in Figure 1) and 'Dual Shell Flashes' (DSFs, early TPAGB, blue triangles in Figure 1). It can be seen in Figure 1 that the carbon yields at $[\mathrm{Fe} / \mathrm{H}]=-3$, for which there are the most observations, cover the upper envelope of the observations quite well. At $[\mathrm{Fe} / \mathrm{H}]=-4$ there is a fair agreement with the observations, although there are less observations to compare with. At $[\mathrm{Fe} / \mathrm{H}]=-5.45$ the yields show somewhat more $\mathrm{C}$ than is observed, however there are only two stars observed at this metallicity. Another interesting feature seen in this figure 


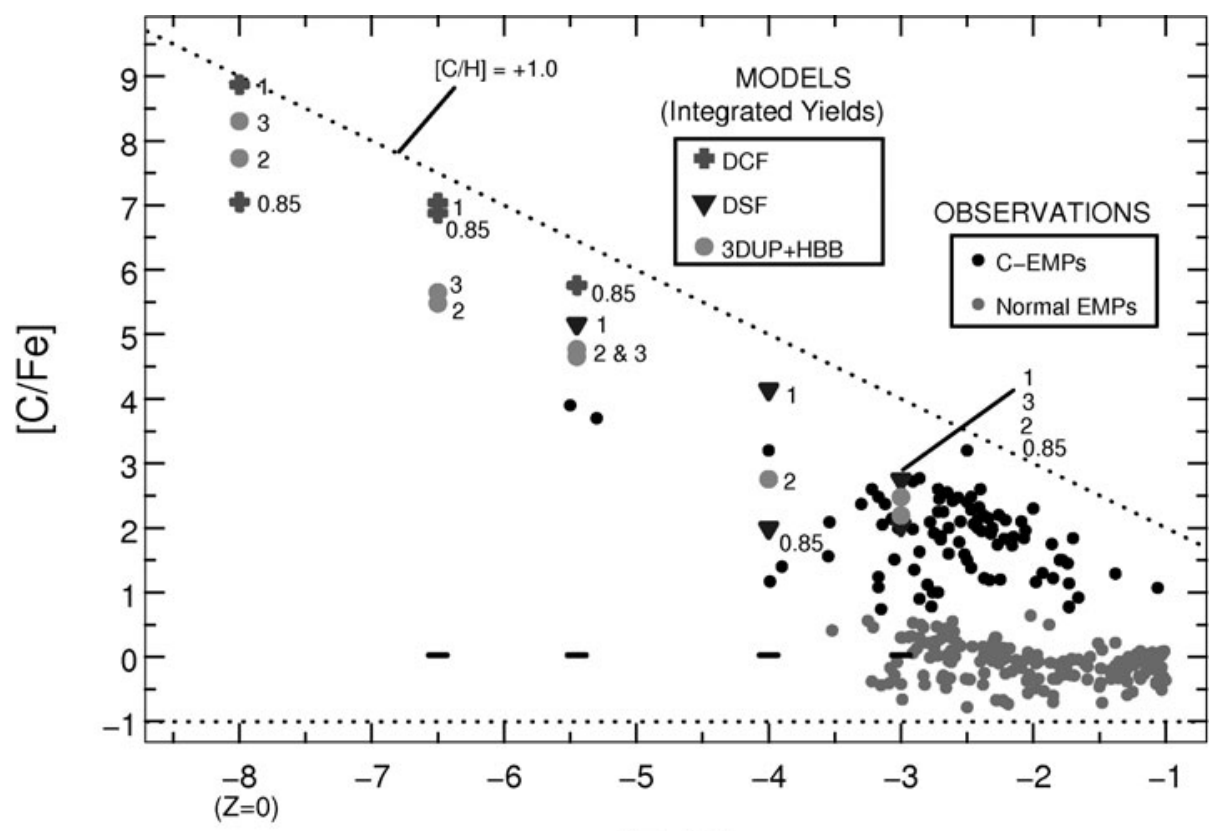

$[\mathrm{Fe} / \mathrm{H}]$

Figure 1. Comparing the carbon yields from all our models with observations of EMP stars. Here we have colour-coded the observations into $[\mathrm{C} / \mathrm{Fe}]$-rich (the CEMPs, black dots) and $[\mathrm{C} / \mathrm{Fe}]$-normal (normal EMPs, grey/magenta dots). We have defined $[\mathrm{C} / \mathrm{Fe}]$-rich by $[\mathrm{C} / \mathrm{Fe}]>+0.7$. It can be seen that the normal EMPs scatter around the Galactic chemical evolution line (at $[\mathrm{C} / \mathrm{Fe}] \sim 0.0)$. The two most Fe-poor stars known, HE 0107-5240 $([\mathrm{Fe} / \mathrm{H}]=-5.3$, Christlieb et al. 2004) and HE 1327-2326 $([\mathrm{Fe} / \mathrm{H}]=-5.4$, Aoki et al. 2006) are labelled. We note that the most C-rich stars have similar amounts of carbon as the Sun, giving them a Z-defined metallicity that is roughly solar. Observational data sets are from Frebel et al. (2006), Spite et al. (2006), Aoki et al. (2007), Beers et al. (2007), and Cohen et al. (2006). The short horizontal lines indicate the starting composition of the models (in this case they are all at $[\mathrm{C} / \mathrm{Fe}] \sim 0$, except for the $Z=0$ models). We have plotted the $Z=0$ model yields at $[\mathrm{Fe} / \mathrm{H}]=-8$ for comparison. An upper envelope to the self-pollution of the models - and the observations - is marked by the dotted line at $[\mathrm{C} / \mathrm{H}]=+1.0$. The yields from our models are colour- and shapecoded to highlight the different episodes that produced the bulk of the pollution in each yield (see text for details). Numbers beside each yield marker indicate initial stellar mass, in $\mathrm{M}_{\odot}$.

is that the model yields predict $[\mathrm{C} / \mathrm{Fe}]$ to continue increasing towards lower metallicities. Furthermore, taking into account the evolutionary stage at which the surface pollution is gained in the lower mass models $\left(M=0.85,1.0 M_{\odot}\right)$ - ie. the DCF events rather than the AGB - the models also predict a higher proportion of C-rich stars at lower and lower metallicities. This is due to the fact that these stars already have self-polluted surfaces during the HB stage - which has a lifetime roughly 1 order of magnitude longer than the AGB phase. We note that, although the lifetimes of all but the least massive models in our grid $\left(M=0.85 M_{\odot}\right)$ are too short to be still 'living' today, the more massive stars could still have contributed to the chemical enrichment of the CEMP population through binary mass transfer (see eg. Suda et al. 2004).

Last but not least we stress that although the $[\mathrm{C} / \mathrm{Fe}]$ abundances in the yields compare reasonably with the observations, a 'multi-element' comparison is a much more stringent test of the nucleosynthesis results. We shall make comparisons with the available observed abundance patterns in our upcoming papers. 


\section{Some Sources of Uncertainties}

\subsection{Mass Loss Rates at $Z=0$ and Very Low Metallicity}

A key problem with modelling EMP giant stars is the unknown driver(s) of mass loss. Currently the dominant theory is that mass is lost from red giant envelopes through radiation pressure acting on grains. Thus, in the EMP or $Z=0$ regime, mass loss is thought to be negligible. However, due to the peculiar evolution of models of EMP and $Z=0$ stars, we argue that mass loss is significant in AGB stars at these metallicities. In fact for this study we decided to retain the standard treatment for mass loss (the MONSTAR code uses empirical mass loss formula from Reimers 1975 during the RGB and that of Vassiliadis \& Wood 1993 during the AGB), for the following reasons.

The first reason is related to the fact that the RGB phases of $Z=0$ and EMP models are very short (or non-existent - many of our stars stay blue until the AGB). This is because He is ignited much earlier in EMP stars than in stars of comparable mass at higher metallicities. For the low-mass EMP models that do make it to the core He flash, the luminosity at the tip of the RGB can be up to 1 order of magnitude lower than in Solar metallicity models (also see eg. D'Antona 1982). Since mass loss is strongly dependent on luminosity the RGB mass loss in these stars is thus largely negligible, particularly at the lowest metallicities (although this is less true for $[\mathrm{Fe} / \mathrm{H}] \gtrsim-4.0$ in terms of the yields this material only has a diluting effect). Therefore the choice of mass-loss formalism is not very important on the EMP RGB, and it is the AGB mass loss that we need to follow properly.

The second reason is that, as mentioned in the Section 3, all these models experience some sort of surface polluting episode - be it from dredged-up material from the DCF or DSF events, or through normal TDU events. Moreover, the Dual Flashes cause pollution before or at the very beginning of the AGB phase, so the surface is polluted for the whole of the AGB. Surface pollution via TDU in the higher mass models occurs over a longer timescale, but we note that the majority of the mass loss occurs towards the end of the AGB, when the luminosity is high and the surface is strongly polluted. All of this has the consequence that the surface of the AGB models usually have metallicities approaching that of the LMC or even Solar (as defined by $Z=1-X-Y$ rather than Fe - they are still metal poor in terms of Fe). Thus, since the stellar surfaces have (some of) the ingredients needed to form grains, we argue that using the standard mass loss formula given by Vassiliadis \& Wood (1993) is warranted. We also note that metallicity is also indirectly taken into account by the mass loss formulae, since they depend on bulk stellar properties (such as radius, luminosity, pulsation period), which vary significantly with metallicity.

Although we have argued that the mass loss in these EMP AGB stars should be comparable to that of higher metallicity stars, we also suggest that the mass loss should be handled better in future models. This requires observations of the mass-loss of metal-poor stars, as well as improved theoretical models. Some work in the observational direction has recently been reported by Origlia et al. (2002) and McDonald \& van Loon (2007). Both studies derive mass-loss rates from observations of a selection of globular clusters covering a wide metallicity range $(\sim-2.2<[\mathrm{Fe} / \mathrm{H}]<-0.6)$. Interestingly both studies find no dependence of mass-loss on metallicity, although more work is probably needed to confirm this. On the theoretical front Mattsson et al. (2008) report that mass loss may be more dependent on pulsation than metallicity, and as such low metallicity stars may still have high mass-loss rates. 


\subsection{The Dual Flash Events $\&$ the Need for Multidimensional Fluid Dynamics Simulations}

As mentioned in Section 3 models of EMP and $Z=0$ stars undergo violent evolutionary episodes involving proton ingestion into hot He burning regions. The most severe of these events is the Dual Core Flash. It occurs in low-mass stars with metallicity $[\mathrm{Fe} / \mathrm{H}] \lesssim-2.5$ (Fujimoto et al. 2000). When the proton-rich material is mixed down into the hot Heflash-driven convective zone it burns at a very high rate. This flash reaches luminosities comparable to the core He flash itself but its onset is much more sudden than that of the core He flash. The rapid onset and short timescale of the H-flash requires high spatial and temporal resolution to simulate. For instance, the time steps in our models during these phases are often as low as $\sim 10^{-3}$ years (sometimes shorter). Such short time steps violate the assumption of hydrostatic equilibrium implicit in our code and thus introduce an uncertainty in our modelling. We are however unsure whether this has any significant effect in this case, but suggest that multidimensional fluid dynamics calculations are needed to check this. Other uncertainties during this challenging phase of evolution have been explored by Schlattl et al. (2001) who performed some tests (using a 1D stellar code) and found that the initial He abundance and atomic diffusion can effect the occurrence of the DCFs. Another argument for the necessity of fluid dynamics calculations arises from the likely inadequacy of the Mixing Length Theory for approximating convection in this regime of high nuclear energy release and turbulent convection (indeed, it was not designed for these conditions). Following these events properly is very important for the resulting yields of these stars, since in many cases it is these events (and the associated mixing up of the processed material) that dominate the pollution of the envelope (although at higher masses the TDU tends to dominate). We note that, since the timescale of evolution for a H-flash is of order years, it is still impossible to follow the entire evolution of the event with current multidimensional fluid dynamics codes. However even following a few hours of the evolution may reveal important features - such as if the $\mathrm{H}$ and He convection zones remain separated or merge. We are currently attempting these simulations thanks to new collaborations formed at this IAU Symposium.

\subsection{Other Uncertainties}

Other uncertainties that may effect the yields of our models include:

- No rotation included: EMP/Pop III stars are more compact, so they should rotate faster. This may cause extra mixing and effect the yields.

- Nuclear reaction rates are known to contain uncertainties (although some are well constrained). Varying reaction rates within their expected uncertainties can give us an idea of how uncertain the yield is for each species.

- Low temperature opacities used in our model (and most stellar codes) do not include the opacity effects of enhanced carbon (or nitrogen) on the cool AGB surfaces. This has feedback effects that can significantly alter the evolution and therefore the yields (see eg. Marigo 2002, Lederer \& Aringer 2008, Cristallo et al. 2007).

\section{Conclusion}

Our study gives a 'zeroth order' set of models and yields for low mass, EMP stars. Although the models can produce the large amounts of carbon needed to explain the CEMPs, we need to compare our yield results with the observed abundance patterns for a range of species. As discussed the models are naturally subject to many uncertainties, all of which need to be investigated for future modelling. Finally we stress again that fluid 
dynamics simulations are urgently needed to assess he properties of the proton ingestion flashes and how this affects the further evolution and yields.

\section{Acknowledgements}

We utilised the APAC supercomputer for this work (project g61). We would like to thank the organisers of this IAU symposium held in Sanya, China.

\section{References}

Fujimoto, M. Y., Iben, I. J., \& Hollowell, D. 1990 ApJ 349, 580

Cassisi, S., Castellani, V., \& Tornambe, A. 1996 ApJ 459, 298

Siess, L., Livio, M., \& Lattanzio, J. 2002 ApJ 570, 329

Schlattl, H., Salaris, M., Cassisi, S., \& Weiss, A. 2002 A\&A 395, 77

Picardi, I., Chieffi, A., Limongi, M., Pisanti, O., Miele, G., Mangano, G., \& Imbriani, G. 2004 ApJ 609, 1035

Suda, T., Aikawa, M., Machida, M. N., Fujimoto, M. Y., \& Iben, I. J. 2004 ApJ 611, 476

Schlattl, H., Cassisi, S., Salaris, M., \& Weiss, A. 2001 ApJ, 559, 1082

Beers, T. C. \& Christlieb, N. 2005 ARA\&A 43, 531

Shigeyama, T. \& Tsujimoto, T. 1998 ApJL 507, L135

Limongi, M., Chieffi, A., \& Bonifacio, P. 2003 ApJL 594, L123

Fujimoto, M. Y., Ikeda, Y., \& Iben, I. J. 2000 ApJ 529, L25

Weiss, A., Schlattl, H., Salaris, M., \& Cassisi, S. 2004 A\&A 422, 217

Christlieb, N., Gustafsson, B., Korn, A. J., Barklem, P. S., Beers, T. C., Bessell, M. S., Karlsson, T., \& Mizuno-Wiedner, M. 2004 ApJ 603, 708

Aoki, W., Frebel, A., Christlieb, N., Norris, J. E., et al. 2006 ApJ 639, 897

Frebel, A. Christlieb, N., Norris, J. E. , Beers, T. C., et al. 2006 ApJ 652, 1585

Spite, M., Cayrel, R., Hill, V., Spite, F., et al. 2006 A\&A 455, 291

Aoki, W., Beers, T. C., Christlieb, N., Norris, J. E., Ryan, S. G., \& Tsangarides, S. 2007 ApJ 655,492

Beers, T. C., Sivarani, T., Marsteller, B. , Lee, Y., Rossi, S., \& Plez, B. 2007 AJ 133, 1193

Cohen, J. G., McWilliam, A., Shectman, S. , Thompson, I., Christlieb, N., Melendez, J., Ramirez, S., Swensson, A., \& Zickgraf, F.-J. 2006 AJ 132, 137

Frost, C. A. \& Lattanzio, J. C. 1996 ApJ 473, 383

Wood, P. R. \& Zarro, D. M. 1981 ApJ 247, 247

Iglesias, C. A. \& Rogers, F. J. 1996 ApJ 464, 943

Ferguson, J. W., Alexander, D. R., Allard, F., Barman, T., Bodnarik, J. G., Hauschildt, P. H., Heffner-Wong, A., \& Tamanai, A. 2005 ApJ 623, 585

Meynet, G., Maeder, A., \& Mowlavi, N. 2004 A\& A 416, 1023

Cannon, R. C. 1993 MNRAS 263, 817

Lattanzio, J., Frost, C., Cannon, R., \& Wood, P. R. 1996 MmSAI 67, 729

Lugaro, M., Ugalde, C., Karakas, A. I., Görres, J., Wiescher, M., Lattanzio, J. C., \& Cannon, R. C. 2004 ApJ 615, 934

Reimers, D. 1975 Memoires of the Societe Royale des Sciences de Liege 8, 369

Vassiliadis, E. \& Wood, P. R. 1993 ApJ 413, 641

D'Antona, F. $1982 A \xi A$ 115, L1

Origlia, L., Rood, R. T., Fabbri, S., Ferraro, F. R., Fusi Pecci, F., \& Rich, R. M. 2007 ApJL $667, \mathrm{~L} 85$

Mattsson, L., Wahlin, R., Höfner, S., \& Eriksson, K. 2008 A $\mathscr{G} A$ 484, L5

McDonald, I. \& van Loon, J. T. 2007 A $E A$ 476, 1261

Origlia, L., Ferraro, F. R., Fusi Pecci, F., \& Rood, R. T. 2002 ApJ 571, 458

Marigo, P., Girardi, L., Chiosi, C., \& Wood, P. R. 2002 A\& A 371, 152

Cristallo, S., Straniero, O., Lederer, M. T., \& Aringer, B. 2007 ApJ 667, 489

Lederer, M. T. \& Aringer, B. 2008 in: Evolution and Nucleosynthesis in AGB Stars, American Institute of Physics Conference Series, 1001, 11 


\section{Discussion}

WALDMAN: Is the mixing of hydrogen into the helium burning layer a consequence of using the Schwarzschild criterion rather then Ledoux?

CAmpBell: The main factor seems to be that the He ignition occurs so far off-center, close to the $\mathrm{H}-\mathrm{He}$ discontinuity. The weaker entropy barrier in $Z=0$ and extremely metal-poor stars also plays a role. I don't know if using the Ledoux criterion would be important or not. 